\title{
Optimal Designing of the Rod Structure
}

\author{
Klyuev S.V. ${ }^{1}$, Klyuev A.V. ${ }^{2}$, Petrov N.I. ${ }^{3}$, Promahov V.V. ${ }^{4}$, Klimenko V.A. ${ }^{5}$ \\ ${ }^{l}$ Candidate of Engineering Sciences (Ph.D.), Associate Professor, Belgorod State Technological University named after V.G. Shukhov, \\ ${ }^{2}$ Candidate of Engineering Sciences (Ph.D.), Associate Professor, Belgorod State Technological University named after V.G. Shukhov, \\ ${ }^{3}$ Moscow State University of Civil Engineering (National Research University), D. Mendeleev University of Chemical Technology of Russia,
}

${ }^{4}$ Ph.D, National Research Tomsk State University,

${ }^{5}$ Siberian Center for Industrial Design and Prototyping (SDC), National Research Tomsk State University

\begin{abstract}
Calculus of variations is a powerful tool for research in the field of mechanics. Variation principles, on the one hand, have a deep theoretical significance, revealing the energy basis of the theory and establishing a connection between different approaches to solving problems. On the other hand, they are of great practical significance, because if there are expressions for functional, they allow to find differential equations and natural (not caused by external circumstances) boundary conditions in cases where it is difficult to do so directly. Moreover, direct methods allow getting a solution to the variation problem, passing the compilation and solution of differential equations. Based on the variation principle of structural synthesis, the design problem for a rod spatial structure is solved. The example of designing a four-rod truss is considered.
\end{abstract}

Keywords: variation principles, optimal designing, structures, method

\section{INTRODUCTION}

Rod spatial structures are widely used in the construction industry of the national economy. First of all, we should name television towers, power transmission towers, and antennas. The work of Soviet and foreign scientists was aimed at creating methods for calculating such structures. However, these methods do not provide for modern design tasks based on a comprehensive coverage of material resources by varying their composition and the shape of the structure itself [1-8].

They were implemented for optimal design of certain types of civil and industrial structures of the tower type. This is an urgent problem, the solution of which will lead to a significant economic effect. At the same time, the design solution will not create additional difficulties in the technological processes for the construction of the considered type of structures.

Designing calculations imply optimal shape and optimal combinations of materials. The considered structures have a rod framework and usually are statically indeterminate systems. With a small number of rods, classical methods for calculating them can be used - the forces method and the displacement method. As the number of rods increases, these methods provide a more complex solution, so designers more often use the finite element method, namely, its variant of the displacement method [9-16].

The design calculation can be made in the form of an iterative process based on analytical calculation and parameter correction. However, it may happen that the convergence of this process requires a large number of cycles. The sensitivity analysis undertaken to get out of this situation is complex for design engineers. Therefore, new approaches to optimizing structures based on analogies with the evolution of natural systems and organisms are currently being proposed. These are so-called evolutionary and genetic algorithms.

Their origin occurred in the last two decades of the last century in foreign design practice. In our country, they have not received sufficient development yet. This is partly due to the fact that the applied criteria for optimal design (minimum volume, minimum weight) are not combined with the principles of development of natural organisms. The old criteria have been replaced by the energy criterion of rational design recently. It is based on the general physical principle of stationary action, which is relevant to all material objects.

Thus, the introduction of the energy criterion and evolutionary and genetic algorithms into the calculation practice creates a coherent scheme for obtaining the optimal project [17-23]. Evolutionary algorithms have been used in our country in the study of thermodynamic processes. In the construction business, their use is in its infancy.

Evolutionary processes (heredity, mutation, and selection) are transformed in the development stages of the project: initial data, correction of individual parameters during the project calculation, selection of the best option based on the energy criterion. To implement this process, the appropriate software is required for using high-speed computers, which allows solving the tasks set effectively.

\section{MATERIALS AND RESEARCH METHODS}

The solution of the problem is constructed as a sequence of using one or more operators. Each of them involves generating a number of solutions and selecting one of them based on an energy criterion. Operators differ in the level 
(specification of solutions) and cost of their implementation.

The formulation of the volume minimization problem is strictly consistent with the principle of stationary action only under additional conditions in the form of integral connections with energy point.

The operator that is applied to topology in order to obtain geometry is directly related to directory constraints, such as the dimensions of the structure, as well as the nature and location of the load. In the case of a tower, the load is concentrated in the nodes.

We can assume many possible variants of the system, which become the starting point for an evolutionary strategy for solving a specific problem. However, their number is reduced when using variation principles of structural synthesis.

The project task is usually solved under additional conditions that impose restrictions on the desired parameters.

\section{RESULTS AND DISCUSSION}

The isoperimetric problem of forming a structure from homogeneous material is solved for given volume $V_{0}$. The Castigliano functional for a rod system has the form:

$I=\sum_{i=1}^{n} \frac{N_{i}^{2} l_{i}}{2 E A_{i}}+\mu_{1}\left(\sum_{i=1}^{n} A_{i} l_{i}-V_{0}\right)$

where $N_{i}$ - longitudinal force in the $i-\operatorname{rod}, n$ - number of rods; $l_{i}$ and $A_{i}$ - the length and cross-sectional area of the $i-$ rod, respectively; $E$ - longitudinal elasticity modulus; $\mu_{1}$ - Lagrange multiplier.

The consequence of the stationarity of the functional is the $m$ compatibility equations of deformations $(m-$ the number of extra connections)

$\partial I / \partial N_{m}=0$,

the volume equation

$\sum_{i=1}^{n} A_{i} l_{i}=V_{0}$

and $r$ structure formation equations $(r-$ the number of variable parameters); in particular, at varying the angles $\alpha_{i}$ that determine the geometry of the structure, we have

$\partial I / \partial \alpha_{i}=0$.

At varying the cross-section areas of the stretched rods, we get

$N_{i}^{2} /\left(2 E A_{i}^{2}\right)=\mu_{1}(=$ const $)$.

In rod systems with compressed rods, the condition for safe stability must be met. This is equivalent to introducing a virtual state with internal forces $N_{i} / \varphi_{i}$ for compressed rods
( $\varphi_{i}$-coefficient of reduction of the design resistance). Thus, by analogy with formula (5), we obtain the equation

$N_{i}^{2} /\left(2 E \varphi_{i}^{2} A_{i}^{2}\right)=\mu_{1}=($ const $)$.

As an example, we consider the design of a four-rod truss (see fig.1). Let us determine the angle $\alpha$ and cross-section areas of rods 1,2 and 3 . Based on the presented analysis, we can assume that rod 1 is stretched, and rods 2 and 3 are compressed.

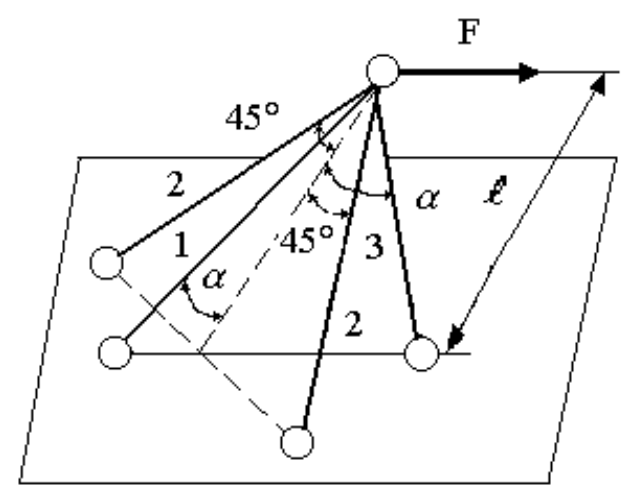

Fig. 1. Four-rod truss

Taking into account the signs of force, we find

$N_{1}=\frac{F}{\sin \alpha}-N_{3}, \quad N_{2}=\frac{1}{\sqrt{2}}\left(F \operatorname{ctg} \alpha-2 N_{3} \cos \alpha\right)$

and write the functional (1) as

$$
\begin{aligned}
I= & \frac{1}{2 E}\left[\frac{\left(F / \sin \alpha-N_{3}\right)^{2} l}{A_{1} \cos \alpha}+\frac{2\left(F \operatorname{ctg} \alpha-2 N_{3} \cos \alpha\right)^{2} l \sqrt{2}}{\varphi_{2}^{2} A_{2}}+\frac{N_{3}^{2} l}{\varphi_{3}^{2} A_{3} \cos \alpha}\right]+ \\
& +\mu_{1}\left(A_{1} \frac{l}{\cos \alpha}+2 A_{2} l \sqrt{2}+A_{3} \frac{l}{\cos \alpha}-V_{0}\right)
\end{aligned}
$$

6 equations of the type (2), (3), (4), (5), (6) follow from the stationary condition of the functional (7):

$$
\begin{aligned}
- & \left(F-N_{3} \sin \alpha\right) \varphi_{2}^{2} \varphi_{3}^{2} A_{2} A_{3} \\
& -2 \sqrt{2}\left(F \operatorname{ctg} \alpha-2 N_{3} \cos \alpha\right) \varphi_{3}^{2} A_{1} A_{3} \sin \alpha \cos \alpha+ \\
& +N_{3} \varphi_{2}^{2} A_{1} A_{2} \sin \alpha=0
\end{aligned}
$$

$$
A_{1} l+2 \sqrt{2} A_{2} l \cos \alpha+A_{3} l-V_{0} \cos \alpha=0
$$

$$
\begin{aligned}
& \frac{1}{2 E}\left[\frac{2\left(F / \sin \alpha-N_{3}\right) F\left(-\cos \alpha / \sin ^{2} \alpha\right) \cos \alpha+\left(F / \sin \alpha-N_{3}\right)^{2} \sin \alpha}{A_{1} \cos ^{2} \alpha}+\right. \\
& \left.+\frac{4 \sqrt{2}\left(F \operatorname{ctg} \alpha-2 N_{3} \cos \alpha\right)\left(-F / \sin ^{2} \alpha+2 N_{3} \sin \alpha\right)}{\varphi_{2}^{2} A_{2}}+\frac{N_{3}^{2} \sin \alpha}{\varphi_{3}^{2} A_{3} \cos ^{2} \alpha}\right]+
\end{aligned}
$$


International Journal of Engineering Research and Technology. ISSN 0974-3154, Volume 13, Number 11 (2020), pp. 3757-3760

(C) International Research Publication House. https://dx.doi.org/10.37624/IJERT/13.11.2020.3757-3760

$+\mu_{1}\left(A_{1}+A_{3}\right)\left(\sin \alpha / \cos ^{2} \alpha\right)=0$

$$
\begin{aligned}
& -\frac{\left(F / \sin \alpha-N_{3}\right)^{2}}{2 E A_{1}^{2}}+\mu_{1}=0 ; \\
& -\frac{\left(F \operatorname{ctg} \alpha-2 N_{3} \cos \alpha\right)^{2}}{2 E \varphi_{2}^{2} A_{2}^{2}}+\mu_{1}=0 ; \\
& -\frac{N_{3}^{2}}{2 E \varphi_{3}^{2} A_{3}^{2}}+\mu_{1}=0 .
\end{aligned}
$$

The solution of the system of equations (8) - (13) allows finding several combinations of parameters $\alpha, A_{1}, A_{2}$ and $A_{3}$, which satisfy the condition of stationarity of the functional (7), but only one option determines the rational design, which is revealed in the selection process.

It may happen that among the obtained combinations of parameters there are some that contradict the previously accepted signs of forces. This leads to an unjustified introduction or absence of $\varphi$ in the formula (7). Correction of the preliminary signs of forces in the rods can be attributed to the mutation operation. The new system of equations of type (8) - (13) will give another series of solutions.

The purpose of selection is to identify a combination of parameters in series of solutions that corresponds to the minimum strain energy. We can also say that the optimal design option is the system with the greatest rigidity, as it corresponds to the minimum work of external forces.

At $F=20 \mathrm{\kappa H}$ and $E=2 \cdot 10^{5} \mathrm{M \Pi l}$ the rational project contains: $\alpha=1,11$ рад; $A_{1}=0,0001014 \mathrm{M}^{2}$;

$A_{2}=0,0000705 \mathrm{M}^{2} ; A_{3}=0,0000233 \mathrm{M}^{2}$;

$N_{3}=4,66$ кH.

Based on the stress values and the accepted modulus of longitudinal elasticity, we select a material with the necessary strength.

We calculate the force modules in the rods 1 and 2:

$$
\begin{aligned}
N_{1} & =\frac{F}{\sin \alpha}-N_{3}=\frac{20}{0,896}-4,66=17,66 \kappa \mathrm{H}, \\
N_{2} & =\frac{1}{\sqrt{2}}\left(F \operatorname{ctg} \alpha-2 N_{3} \cos \alpha\right) \\
& =\frac{1}{\sqrt{2}}(20 \cdot 0,497-2 \cdot 4,66 \cdot 0,445)=1,87 \kappa \mathrm{H} .
\end{aligned}
$$

In this case, the stresses are as follows:

$$
\begin{aligned}
& \sigma_{1}=N_{1} / A_{1}=(17,66 / 0,0001014) \cdot 10^{-3} \mathrm{M \Pi а}=174,16 \mathrm{M \Pi а} ; \\
& \sigma_{2}=N_{2} / A_{2}=-(1,17 / 0,0000705) \cdot 10^{-3} \mathrm{M \Pi a}=-16,6 \mathrm{M \Pi а} ; \\
& \sigma_{3}=N_{3} / A_{3}=-(4,66 / 0,0000233) \cdot 10^{-3} \mathrm{M \Pi а}=-200 \mathrm{M \Pi а} ;
\end{aligned}
$$

The consistent strength can be provided by choosing a material in the form of steel S235, for which the yield strength is $225 \mathrm{MPa}$.

\section{CONCLUSIONS}

The design calculation is completed by calculating the dimensions of the cross sections of the rods. For stretched rods, they are determined directly from the formula that relates the area and cross-section parameters.

For compressed rods, in addition to this formula, the formula is used that relates the radius of inertia and the cross-section parameters. We will perform these calculations for $\operatorname{rod} 3$, assuming for it a tubular section with an outer diameter $d_{e}$ and an inner diameter $d_{i}$. Let us calculate the radius of inertia:

$i=(\mu l) / \lambda$,

where $\mu-$ the coefficient of the given length, which in this case is equal to $1 ; \lambda$ - flexibility of the rod.

At the received coefficient $\varphi=0,75$ for selected material $\lambda=77$. Therefore, $i=(300 / 0,445) / 77=8,76 \mathrm{~cm}$.

Thus, to find $d_{e}$ and $d_{i}$, we have two equations

$$
\begin{aligned}
& \frac{\pi}{4}\left(d_{e}^{2}-d_{i}^{2}\right)=0,233 ; \\
& \frac{1}{4} \sqrt{d_{e}^{2}+d_{i}^{2}}=8,76 .
\end{aligned}
$$

As a result of solving the system of equations we get the values of diameters: $d_{e}=24,9 \mathrm{~cm}, d_{i}=24,6 \mathrm{~cm}$.

\section{REFERENCES}

[1] Vatin, N.I., Nazmeeva, T., \& Guslinscky, R. (2014). Problems of cold-bent notched c-shaped profile members. Advanced Materials Research, 941-944, 18711875.

[2] Garifullin, M., Vatin, N., Jokinen, T., \& Heinisuo, M. (2017). Numerical solution for rotational stiffness of RHS tubular joints. Advances and Trends in Engineering Sciences and Technologies II - Proceedings of the 2nd International Conference on Engineering Sciences and Technologies, ESaT 2016, 81-86.

[3] Manzhilevskaya, S.E. (2019). Organizational and economic problems of ecological safety in construction. Construction Materials and Products, 2(4), 73 -78.

[4] Evdokimov, P.A. (2019). Features of assessment of anthropogenic loads on natural complexes. Construction Materials and Products, 2(5), $19-24$.

[5] Tinkov, D.V. (2015). Comparative analysis of analytical solutions to the problem of truss structure deflection. Magazine of Civil Engineering, 57(5), 66-73. 
International Journal of Engineering Research and Technology. ISSN 0974-3154, Volume 13, Number 11 (2020), pp. 3757-3760

(C) International Research Publication House. https://dx.doi.org/10.37624/IJERT/13.11.2020.3757-3760

[6] Petrosov, D. A., Lomazov, V. A., Mironov, A. L., Klyuev, S. V., Muravyov, K. A., \& Vasilievna, F. M. (2018). Intellectual structural-parametric synthesis of large discrete systems with specified behavior. Journal of Engineering and Applied Sciences, 13(8), 21772182.

[7] Klyuev, S.V., Klyuev, A.V., Grishko, A.K., Trukhanov, S.V. (2019). Management of the design parameters in optimal design problems. Materials Science Forum, 974, $723-728$.

[8] Tinkov, D.V. (2016). The optimum geometry of the flat diagonal truss taking into account the linear creep. Magazine of Civil Engineering, 61 (1), 25-32.

[9] Kirsanov, M.N. (2015). Analysis of the deflection of a strut-type lattice girder truss. Magazine of Civil Engineering, 57(5), 58-65.

[10] Mailyan, L.R., Yazyev, S.B., Sabitov, L.S., Konoplev, Yu.G., Radaykin, O.V. (2019). Stress-strain state of the system "Combined tower - reinforced concrete foundation-foundation soil" of high-rise structures. Construction Materials and Products, 2(6), 29 - 37.

[11] Deryushev, V.V., Zaitseva, M.M., Evseev, D.V., \& Kosenko, E.E. (2019). The concentration of thermal stresses in metal materials and construction under local heating. Construction Materials and Products, 2(3), 72 -77 .

[12] Kirsanov, M.N. (2016). Analysis of the buckling of spatial truss with cross lattice. Magazine of Civil Engineering, 64 (4), 52-58.

[13] Alekseytsev, A.V., Kurchenko, N.S. (2017). Deformations of steel roof trusses under shock emergency action. Magazine of Civil Engineering, 73 (5), 3-13.

[14] Klyuev, S.V., Shlychkov, D.I., Muravyov, K.A., Ksenofontova, T.K. (2020). Optimal Design of Building Structures. International Journal of Advanced Science and Technology, 29(5), 2577 - 2583.

[15] Kravchuk, A.S., Kravchuk, A.I., Lopatin, S.N. (2019). Complete soulution of lame problem for a thick-walled composite nonlinearly deformable cylinder. Construction Materials and Products, 2(4), 64 - 72.

[16] Klyuev, S.V., Klyuev, A.V., Abakarov, A.J., Danilov, V.F., Chubenko, E.Ph. (2019). Optimization of building constructions on the basis of genetic algorithm. Journal of Computational and Theoretical Nanoscience, 16(7), $2950-2953$.

[17] Poloz, M.A., Yasser Garib Sami, Shevchenko, A.V. (2019). Application of stepwise and iterative method in the calculation of bending prestressed precast and monolithic elements taking into account the physical nonlinearity. Construction Materials and Products, 2(3), $12-27$.

[18] Serpik, I.N., Alekseytsev, A.V., Balabin, P.Yu., Kurchenko, N.S. (2017). Flat rod systems: Optimization with overall stability control. Magazine of Civil
Engineering, 76 (8), 181-192.

[19] Leontyev, V.V., Kondratova, E.V., Kolomiychenko, V.P. (2019). Investigation of the stress condition of riveted joints by finite element method. Construction $\mathrm{Ma}$ terials and Products, 2(1), $32-36$.

[20] Indeykin, I.A., Chizhov, S.V., Shestakova, E.B., Antonyuk, A.A., Evtukov, E.S., Kulagin, K.N., Karpov, V.V., Golitsynsky, G.D. (2017). Dynamic stability of the lattice truss of the bridge taking into account local oscillations. Magazine of Civil Engineering, 76 (8), 266-278.

[21] Tusnina, O.A. (2018). Finite element analysis of crane secondary truss. Magazine of Civil Engineering, 77 (1), 68-89.

[22] Klyuev, S.V., Abakarov, A.J., Lesovik, R.V., Muravyov, K.A., Tatlyev, R.Dz. (2019). Optimal engineering of rod spatial construction. Journal of Computational and Theoretical Nanoscience, 16(1), 200 - 203.

[23] Yuryev, A. G., \& Zinkova, V. A. (2019). Ata El-Karim Soliman. Truss design calculation, Construction Materials and Products, 2(1), 37-44. 